\title{
Structural Study of Amorphous CoFeB Magnetic Thin Films by Electron Diffraction
}

\author{
A. Kohn, ${ }^{*}$ D. Kirk, ${ }^{*}$ K. B. Borisenko, ${ }^{*}$ C. Lang, ${ }^{*}$ J. Schmalhorst, ${ }^{*}$ G. Reiss, ${ }^{* *}$ and D. J. H. \\ Cockayne* \\ * Department of Materials, University of Oxford, Oxford OX1 3PH, United Kingdom \\ ** Department of Physics, Thin Films and Physics of Nanostructures, Bielefeld University, 33501 \\ Bielefeld, Germany
}

Amorphous materials comprised of transition metals (TM) and metalloid (M) atoms are of technological importance; for example $\mathrm{Co}_{40} \mathrm{Fe}_{40} \mathrm{~B}_{20}(\mathrm{CoFeB})$ is used for ferromagnetic thin film electrodes in tunnel magnetoresistance sensors [1]. These materials are ferromagnetic because exchange interactions are determined by nearest neighbour atoms. Moreover, even though the structure is amorphous, in-plane uniaxial magnetic anisotropy can be induced in $\mathrm{CoFeB}$ in an arbitrary direction by annealing within a magnetic field. These observations are our motivation to characterise the short-range-order of amorphous ferromagnets.

Such structural characterization is achieved using reduced density function, G(r), analysis of electron diffraction patterns [3]. Electron diffraction were recorded on a JEOL JEM-3000F and converted to $\mathrm{G}(\mathrm{r})$ [2]. A typical G(r) result of CoFeB, $15 \mathrm{~nm}$ thick, is shown in Fig. 1, from which the most probable bond-lengths are measured. Table 1 presents the results of these measurements up to the $4^{\text {th }}$ TM-TM neighbour. This analysis demonstrates that short-range ordering exists in this material when compared to a calculation of $\mathrm{CoFeB}$ assuming random packing. Amorphous $\mathrm{CoFeB}$ also has medium-range-order as observed in the peak of the $G(r)$ curve around $6.4 \AA$ while in random packing, no ordering is observed. The overall structure of $\mathrm{CoFeB}$ thin films is further investigated by reverse Monte Carlo (RMC) simulations (see fit to experimental results in Fig. 1), which enable an estimation of the coordination number of the transition-metal components in the CoFeB alloy. In the RMC refined model, distorted polyhedra are discerned with different coordination numbers of the TM, as shown in Fig. 2. Despite an average coordination number of approximately 8 for the TM component, a degree of medium-range order, and a stoichiometry close to CoFe, large local deviations from this octahedral value mean that the structure is not based on bcc-type basic units.

Additionally, the RDF methodology is developed in order to measure the direction-dependent bond length and coordination number in the plane of the film. Directional variations in these parameters are predicted to be the likely origins of induced anisotropy in amorphous ferromagnetic thin films. In this case, the induced anisotropy constant is approximately $2 \mathrm{KJ} / \mathrm{m}^{3}$, which was verified by magnetometry and Lorentz TEM micromagnetic imaging. With nearest-neighbor distances measured to an accuracy of $0.02 \AA$, no variations in the characteristic bond lengths with direction are observed. By studying the coordination numbers, it is shown that a directional ordering effect must be less than $5 \%$ and $1 \%$ for TM-M and TM-TM metal neighbors, respectively.

References

[1] D. D. Djayaprawira, K. Tsunekawa, M. Nagai, H. Maehara, S. Yamagata, N. Watanabe, S. Yuasa, Y. Suzuki, and K. Ando, Appl. Phys. Lett. 86 (2005) 092502.

[2] D. Kirk, A. Kohn, K. B. Borisenko, C. Lang, J. Schmalhorst, G. Reiss, and D. J. H. Cockayne, Phys. Rev. B. 79 (2009) 014203. 
[3] D. J. H. Cockayne, Annu. Rev. Mater. Res. 37 (2007) 159.

[4] A.K. acknowledges the financial support of the Royal Academy of Engineering and the EPSRC-GB.

TABLE 1. Bond lengths in CoFeB thin films.

\begin{tabular}{lccccc}
\hline Bond type & $\mathrm{M}-\mathrm{B}$ & $1^{\text {st }} \mathrm{M}-\mathrm{M}$ & $2^{\text {nd }} \mathrm{M}-\mathrm{M}$ & $3^{\text {rd }} \mathrm{M}-\mathrm{M}$ & $4^{\text {th }} \mathrm{M}-\mathrm{M}$ \\
\hline Bond Length & $2.00 \pm 0.02$ & $2.56 \pm 0.02$ & $4.27 \pm 0.05$ & $5.01 \pm 0.04$ & $6.38 \pm 0.05$
\end{tabular}

$[\AA]$

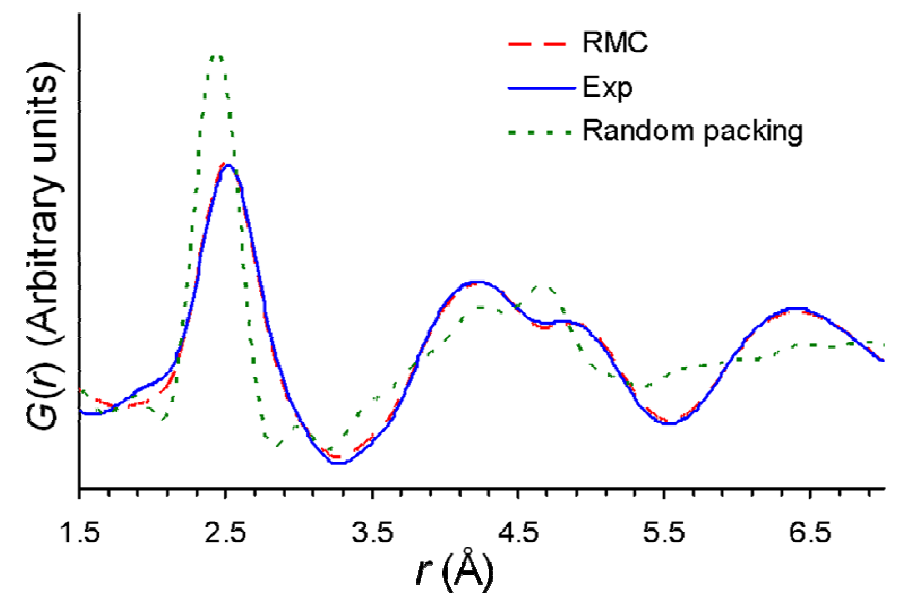

FIG. 1. Comparison of experimental and model reduced density function, $G(r)$, and that obtained from a random packed structure.
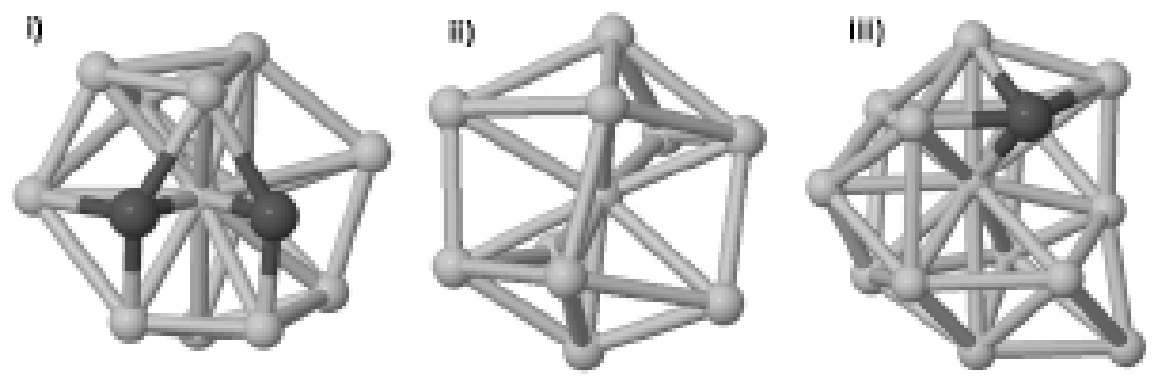

FIG. 2. Structures observed in the refined model structure calculated by RMC.

$\mathrm{TM}$ atoms are light gray and B atoms are dark gray. 